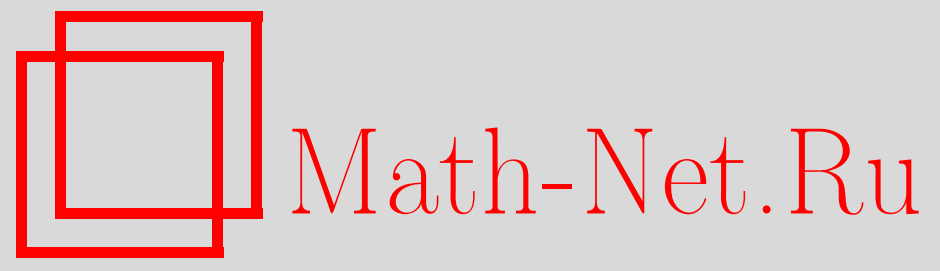

С. С. Марченков, О выразимости функций многозначной логики в некоторых логико-функциональных языках, Дискрет. матем., 1999, том 11, выпуск 4, 110-126

DOI: https://doi.org/10.4213/dm400

Использование Общероссийского математического портала Math-Net.Ru подразумевает, что вы прочитали и согласны с пользовательским соглашением http://www . mathnet.ru/rus/agreement

Параметры загрузки:

IP : 3.85 .5 .30

26 апреля 2023 г., 14:23:23

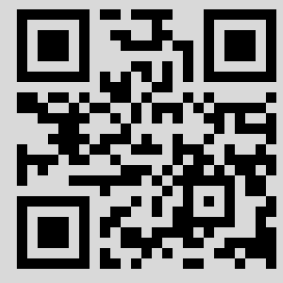




\title{
О выразимости функций многозначной логики в некоторых логико-функциональных языках
}

\author{
(C) 1999 г. $\quad$ С. С. Марченков
}

\begin{abstract}
При любом $k, k \geqslant 2$, для множества функций $k$-значной логики вводятся три логико-функциональных языка: язык позитивной выразимости $\mathrm{Pos}_{k}$, язык первой ступени $1 \mathrm{~L}_{k}$ и язык второй ступени $2 \mathrm{~L}_{k}$. На основе понятия выразимости в языке определяются соответствующие операторы замыкания. Доказывается, что операторы $1 \mathrm{~L}_{k}$-замыкания и $2 \mathrm{~L}_{k}$-замыкания совпадают. $1 \mathrm{~L}_{k}$-замкнутые и $\operatorname{Pos}_{k}$-замкнутые классы характеризуются с помощью симметрических групп и симметрических полугрупп. Выразимость в языках $1 \mathrm{~L}_{k}, \operatorname{Pos}_{k}$ сравнивается с параметрической и термальной выразимостями.

Работа выполнена при поддержке Российского фонда фундаментальных исследований, проект 97-01-00989.
\end{abstract}

\section{1. Введение}

Автор посвящает эту работу светлой памяти Сергея Всеволодовича Яблонского.

Широко распространенным техническим приемом в математике является определение одних объектов через другие. Способы определения могут быть самыми разнообразными: теоретико-множественными, алгебро-логическими, интегро-дифференциальными и другими. В дискретной математике в этих целях в основном применяются алгебро-логические, логико-функциональные и комбинаторные средства. Если рассматривать функции многозначной логики, то известные в литературе способы представления одних функций через другие можно весьма условно разбить на две группы: в первой группе главную роль играют термальные (формульные) представления, во второй группе в основе лежат логические средства.

Для каждого из способов представления формулируется центральная проблема: найти условия, позволяющие для любых функции $f$ и множества функций $F$ решить, представима (выразима) ли функция $f$ через функции множества $F$. Иногда ту же самую проблему формулируют несколько иначе: для любого множества $F$ описать множество всех функций, представимых через функции множества $F$ (замыкание множества $F$ ). В таком виде эта проблема превращается в проблему классификации функций многозначной логики (относительно выбранного способа представления функций). 
Для функций многозначной логики наиболее распространенной является формульная представимость [1-3]. Соответствующая классификационная проблема полностью решена для множества $P_{2}$ булевых функций [4-6]. При $k \geqslant 3$ для множеств $P_{k}$ функций $k$-значной логики решена лишь часть классификационной проблемы, относящаяся к определению предполных классов [1, 7-9]. Кроме того, существует простой алгоритм (см. [1]), который позволяет для произвольных функции $f$ из $P_{k}$ и конечного множества $F$ функций из $P_{k}$ ответить на вопрос, выразима ли функция $f$ формулой через функции множества $F$. Вместе с тем результаты из [10] показывают, что для формульной выразимости функций классификационную проблему при $k \geqslant 3$ вряд ли удастся решить удовлетворительным образом, поскольку возникает континуальное число замкнутых классов.

В последнее время интенсивному исследованию подверглись такие формульные представления, где наряду с любой заданной функцией разрешается также использовать все двойственные (сопряженные) к ней функции, образованные для подстановок из некоторой группы $G$ [11-22]. Оказалось, что если группа $G$ достаточно велика (например, является полной симметрической группой $\mathscr{S}_{k}$ подстановок на множестве $E_{k}$ или ее знакопеременной подгруппой), то соответствующая $G$-классификация конечна. В наиболее важном случае, случае группы $\mathscr{S}_{k}$, удается полностью и с различных позиций охарактеризовать все $\mathscr{S}_{k}$-замкнутые классы и найти в них $\mathscr{S}_{k}$-базисы [11-13, 16-19].

Гораздо меньше исследований посвящено представлениям, где основу составляют логические средства. В этом направлении отметим работы А.В. Кузнецова и его учеников [23-25], а также [26-29]. Отправляясь от работы [23], мы хотим рассмотреть вопрос о выразимости функций многозначной логики в некоторых логикофункциональных языках.

Традиционную формульную выразимость функций в $P_{k}$ можно рассматривать как термальную выразимость в языке $0 \mathrm{~L}_{k}$, который содержит предметные переменные и счетное число функциональных констант для обозначения всех функций из $P_{k}$. Никакие логические символы в языке $0 \mathrm{~L}_{k}$ не используются. Для получения более мощных средств выразимости язык $0 \mathrm{~L}_{k}$ необходимо обогатить равенством, логическими связками и кванторами. При определении параметрической выразимости [23] к языку $0 \mathrm{~L}_{k}$ добавляется равенство, конъюнкция и квантор существования. Соответствующий язык мы обозначаем через $\operatorname{Par}_{k}$. Следующий шаг по линии усиления выразимости состоит в добавлении к языку $\operatorname{Par}_{k}$ дизъюнкции. Этот язык мы обозначаем через $\operatorname{Pos}_{k}$. Расширение языка $\operatorname{Pos}_{k}$ за счет отрицания (либо квантора общности) приводит к языку $1 \mathrm{~L}_{k}$, который имеет полную систему логических связок и оба квантора $\exists$ и $\forall$. Наконец, чтобы обозреть границы выразимости в классе логикофункциональных языков, мы определяем язык второй ступени $2 \mathrm{~L}_{k}$, в котором по сравнению с языком $1 \mathrm{~L}_{k}$ имеются дополнительно функциональные переменные и кванторы по функциональным переменным.

Введенные языки образуют возрастающую цепочку

$$
0 \mathrm{~L}_{k} \subset \operatorname{Par}_{k} \subset \operatorname{Pos}_{k} \subset 1 \mathrm{~L}_{k} \subset 2 \mathrm{~L}_{k}
$$

Возникает вопрос, каковы выразительные возможности языков этой цепочки. Необходимая информация о языке $0 \mathrm{~L}_{k}$ приведена выше. Качественные изменения наблюдаются при переходе от языка $0 \mathrm{~L}_{k}$ к языку $\operatorname{Par}_{k}$. В [23] найдены все (их 25) параметрически замкнутые классы булевых функций, в [24] доказано, что число 
параметрически замкнутых классов в $P_{3}$ конечно, в [29] аналогичное утверждение установлено при любом $k, k \geqslant 4$.

В настоящей работе мы изучаем выразимость в языках $\operatorname{Pos}_{k}, 1 \mathrm{~L}_{k}, 2 \mathrm{~L}_{k}$. Доказано (теорема 1), что выразительные возможности языков $1 \mathrm{~L}_{k}$ и $2 \mathrm{~L}_{k}$ совпадают. При любом $k, k \geqslant 2$, в терминах функций с заданной группой автоморфизмов охарактеризованы все $1 \mathrm{~L}_{k}$-замкнутые классы (теоремы 2,3 ). Указана процедура, позволяющая определить большинство $\operatorname{Pos}_{k}$-замкнутых классов, и найдены нижняя и верхняя границы числа этих классов (теорема 5). Полученные результаты показывают, что выразительные возможности языков строго возрастают как при переходе от языка $\operatorname{Par}_{k}$ к языку $\operatorname{Pos}_{k}$, так и при переходе от $\operatorname{Pos}_{k}$ к $1 \mathrm{~L}_{k}$.

\section{2. Основные понятия}

Пусть $k$ - натуральное число, $k \geqslant 2, E_{k}=\{0,1, \ldots, k-1\}, P_{k}-$ множество всех функций $k$-значной логики $[1,2]$, т.е. функций вида $f: E_{k}^{n} \rightarrow E_{k}, n=1,2, \ldots$

Определим язык $0 \mathrm{~L}_{k}$. Исходными символами языка $0 \mathrm{~L}_{k}$ являются предметные переменные $x_{1}, x_{2}, \ldots$, символы $f_{i}^{(n)}$ для обозначения $n$-местных функций $k$-значной логики, $1 \leqslant i \leqslant k^{k^{n}}, n=1,2, \ldots$, левая и правая скобки и запятая. Обычным образом вводится понятие терма в языке $0 \mathrm{~L}_{k}$ : если $x_{j_{1}}, \ldots, x_{j_{n}}$ - предметные переменные (не обязательно различные), а $f_{i}^{(n)}$ - символ $n$-местной функции, то $f_{i}^{(n)}\left(x_{j_{1}}, \ldots, x_{j_{n}}\right)$ есть терм; если $t_{1}, \ldots, t_{m}$ - термы либо предметные переменные, а $f_{l}^{(m)}-$ символ $m$-местной функции, то $f_{l}^{(m)}\left(t_{1}, \ldots, t_{m}\right)$ также есть терм. Других термов в языке $0 \mathrm{~L}_{k}$ нет.

По техническим причинам наряду с исходными обозначениями будем использовать также обозначения вида $x, y, z, f^{(n)}, g^{(n)}$, возможно, с нижними индексами. Если число переменных у функции определяется из контекста, то верхний индекс, указывающий на число переменных, будем опускать.

Всякий терм $t$ языка $0 \mathrm{~L}_{k}$ очевидным образом задает некоторую функцию $\varphi$ из $P_{k}$. Если $g_{1}, \ldots, g_{r}-$ все символы функций, входящих в терм $t$, то говорим, что терм $t$ выражает функцию $\varphi$ через функции $g_{1}, \ldots, g_{r}$. (В теории функций многозначной логики термы принято называть формулами, терм, составленный из символов функций $g_{1}, \ldots, g_{r},-$ формулой над множеством функций $\left\{g_{1}, \ldots, g_{r}\right\}$, а функцию, выражаемую термом $t$ через функции $g_{1}, \ldots, g_{r},-$ функцией, реализуемой формулой $t$ над множеством функций $\left\{g_{1}, \ldots, g_{r}\right\}$ (см. $\left.[1,2]\right)$.

Пусть $Q \subseteq P_{k}$. Говорим, что функция $\varphi$ выразима через функции множества $Q$, если существует терм $t$, когорый построен из символов функций $g_{1}, \ldots, g_{r}$, входящих в множество $Q$, и который выражает функцию $\varphi$ через функции $g_{1}, \ldots, g_{r}$. Множество всех функций, выразимых через функции множества $Q$, обозначаем через $0 \mathrm{~L}_{k}[Q]$. Обычно множество $0 \mathrm{~L}_{k}[Q]$ называют замыканием $Q$ относительно операции суперпозиции $[1,2]$.

Перейдем к определению языка $\operatorname{Par}_{k}$, языка параметрической выразимости (ср. c [23]). $\mathrm{K}$ исходным символам языка $0 \mathrm{~L}_{k}$ добавляем знак равенства $=$, логическую связку конъюнкция \& и квантор существования Э. Понятие терма в языке $\operatorname{Par}_{k}$ отличается от соответствующего понятия в языке $0 \mathrm{~L}_{k}$ только тем, что в языке $\operatorname{Par}_{k}$ любая предметная переменная также считается термом. Если $t_{1}, t_{2}$ - термы языка $\operatorname{Par}_{k}$, то выражение $\left(t_{1}=t_{2}\right)$ называется элементарной формулой. Из элементарных формул по обычным логическим правилам определяются остальные формулы языка 
$\operatorname{Par}_{k}$. Именно, если $\Phi_{1}, \Phi_{2}-$ формулы языка $\operatorname{Par}_{k}$, а $x_{i}$ - предметная переменная, то $\left(\Phi_{1} \& \Phi_{2}\right)$ и $\left(\exists x_{i}\right) \Phi_{1}$ - также формулы языка $\operatorname{Par}_{k}$. Понятия свободной и связанной переменных предполагаем известными.

Всякая формула языка $\mathrm{Par}_{k}$ с $m$ свободными переменными очевидным образом определяет некоторое $m$-местное отношение на $E_{k}$. Пусть $Q \subseteq P_{k}, \Phi\left(x_{1}, \ldots, x_{m}\right)$ - формула языка $\operatorname{Par}_{k}$ со свободными переменными $x_{1}, \ldots, x_{m}$, все функциональные символы которой суть обозначения функций из $Q$, и формула $\Phi\left(x_{1}, \ldots, x_{m}\right)$ определяет отношение $\rho\left(x_{1}, \ldots, x_{m}\right)$ на $E_{k}$. В этом случае говорим, что формула $\Phi\left(x_{1}, \ldots, x_{m}\right)$ параметрически выражает отношение $\rho\left(x_{1}, \ldots, x_{m}\right)$ через функции множества $Q$. Отношение $\rho$ называем параметрически выразимым через функции множества $Q$, если существует формула, которая параметрически выражает отношение $\rho$ через функции множества $Q$. Совокупность всех отношений, параметрически выразимых через функции множества $Q$, обозначаем через $\operatorname{Par}_{k}[Q]_{R}$. Множества вида $\operatorname{Par}_{k}[Q]_{R}$ называем параметрически замкнутыми классами отношений.

Понятие параметрической выразимости переносится с отношений на функции. Именно, если $\varphi\left(x_{1}, \ldots, x_{m}\right)$ - функция из $P_{k}$, а формула $\Phi\left(x_{1}, \ldots, x_{m}, y\right)$ языка $\operatorname{Par}_{k}$ параметрически выражает отношение $\varphi\left(x_{1}, \ldots, x_{m}\right)=y$ через функции множества $Q$, то говорим, что формула $\Phi$ параметрически выражает функцию $\varphi$ через функции множества $Q$. Совокупность всех функций, параметрически выразимых через функции множества $Q$, обозначаем через $\operatorname{Par}_{k}[Q]_{F}$ и называем параметрическим замыканием множества $Q$. Множества вида $\operatorname{Par}_{k}[Q]_{F}$ называем параметрически замкнутыми классами функций.

Язык $\operatorname{Pos}_{k}$ является расширением языка $\operatorname{Par}_{k}$ : к исходным символам языка $\operatorname{Par}_{k}$ добавляется логическая связка дизъюнкция $\vee$. Понятие терма в языке $\mathrm{Pos}_{k}$ совпадает с понятием терма в языке $\mathrm{Par}_{k}$, а понятие формулы является очевидным обобщением соответствующего понятия для языка $\mathrm{Par}_{k}$. Вместо термина "параметрически выражает" в языке $\operatorname{Pos}_{k}$ применяем термин "позитивно выражает". Множество всех отношений на $E_{k}$ (функций из $P_{k}$ ), которые позитивно выразимы через функции множества $Q$, обозначаем через $\operatorname{Pos}_{k}[Q]_{R}$ (соответственно $\operatorname{Pos}_{k}[Q]_{F}$ ). Множество $\operatorname{Pos}_{k}[Q]_{F}$ называем позитивным замыканием множества $Q$, а множества вида $\operatorname{Pos}_{k}[Q]_{R}\left(\operatorname{Pos}_{k}[Q]_{F}\right)$ позитивно замкнутыми классами отношений (функций).

Язык $1 \mathrm{~L}_{k}$ получается из языка $\operatorname{Pos}_{k}$ добавлением логической связки отрицание $\neg$ и квантора общности $\forall$. Так же, как для языков $\operatorname{Par}_{k}, \operatorname{Pos}_{k}$, вводим понятия "отношение (функция) $1 \mathrm{~L}_{k}$-выразима через функции множества $Q$ ", " $1 \mathrm{~L}_{k}$-замыкание множества $Q$ ” и “ $1 \mathrm{~L}_{k}$-замкнутый класс отношений (функций)”. Множество всех отношений (функций), $1 \mathrm{~L}_{k}$-выразимых через функции множества $Q$, обозначаем через $1 \mathrm{~L}_{k}[Q]_{R}$ (соответственно $1 \mathrm{~L}_{k}[Q]_{F}$ ).

Язык $2 \mathrm{~L}_{k}$ представляет собой язык второй ступени. Исходные символы языка $2 \mathrm{~L}_{k}$ состоят из исходных символов языка $1 \mathrm{~L}_{k}$, к которым добавлены функциональные переменные $F_{i}^{(n)}, i, n=1,2, \ldots$ При определении терма в языке $2 \mathrm{~L}_{k}$ добавляется новый пункт: если $t_{1}, \ldots, t_{n}$ - термы, а $F_{i}^{(n)}$ - функциональная переменная, то $F_{i}^{(n)}\left(t_{1}, \ldots, t_{n}\right)$ - терм. При определении формулы в языке $2 \mathrm{~L}_{k}$ дополнительно разрешаются кванторы по функциональным переменным.

Пусть $\Phi\left(F_{i}^{(n)}\right)$ - формула языка $2 \mathrm{~L}_{k}$ с единственной свободной функциональной переменной $F_{i}^{(n)}$ (в частности, все предметные переменные формулы $\Phi$ связаны). Говорим, что формула $\Phi\left(F_{i}^{(n)}\right)$ определяет функцию $\varphi\left(x_{1}, \ldots, x_{n}\right)$ из $P_{k}$, если $\varphi$ является единственной $n$-местной функцией, удовлетворяющей формуле $\Phi\left(F_{i}^{(n)}\right)$. Как 
для языка $1 \mathrm{~L}_{k}$, определяем понятия “отношение $\rho 2 \mathrm{~L}_{k}$-выразимо через функции множества $Q$ " и " $2 \mathrm{~L}_{k}$-замкнутый класс отношений". При этом в используемых формулах все функциональные переменные должны быть связанными. При определении аналогичных понятий для функций применяем формулы языка $2 \mathrm{~L}_{k}$, в которых имеется только одна свободная переменная, функциональная переменная.

Легко видеть, что всякий параметрически замкнутый класс функций замкнут относительно операции суперпозиции. В самом деле, если терм $t$ языка $0 \mathrm{~L}_{k}$ выражает функцию $\varphi$ через функции $g_{1}, \ldots, g_{r}$, а переменная $y$ не входит в терм $t$, то формула $(t=y)$ языка $\operatorname{Par}_{k}$ параметрически выражает функцию $\varphi$ через функции множества $\left\{g_{1}, \ldots, g_{r}\right\}$.

Поскольку язык $\operatorname{Pos}_{k}$ есть расширение языка $\operatorname{Par}_{k}$, а язык $1 \mathrm{~L}_{k}-$ расширение языка $\operatorname{Pos}_{k}$, всякий позитивно замкнутый класс отношений (функций) является параметрически замкнутым, а всякий $1 \mathrm{~L}_{k}$-замкнутый класс - позитивно замкнутым. Аналогичные утверждения справедливы и для языков $2 \mathrm{~L}_{k}, 1 \mathrm{~L}_{k}$. Для $2 \mathrm{~L}_{k-}$ замкнутых и $1 \mathrm{~L}_{k}$-замкнутых классов отношений это непосредственно следует из определений, так как понятие отношения, $2 \mathrm{~L}_{k}$-выразимого через функции множества $Q$, аналогично соответствующему понятию в языке $1 \mathrm{~L}_{k}$. Что касается функций, то пусть формула $\Phi\left(x_{1}, \ldots, x_{n}, y\right) 1 \mathrm{~L}_{k}$-выражает функцию $\varphi\left(x_{1}, \ldots, x_{n}\right)$ через функции множества $Q$. Тогда функция $\varphi$ будет $2 \mathrm{~L}_{k}$-выражаться через функции множества $Q$ с помощью формулы

$$
\left(\forall x_{1}\right) \ldots\left(\forall x_{n}\right)(\forall y)\left(\left(F_{1}^{(n)}\left(x_{1}, \ldots, x_{n}\right)=y\right) \equiv \Phi\left(x_{1}, \ldots, x_{n}, y\right)\right),
$$

где эквивалентность 三 стандартным образом определяется через связки $\&, \vee, \neg$.

Таким образом, для произвольного множества $Q, Q \subseteq P_{k}$, имеют место включения

$$
\begin{gathered}
0 \mathrm{~L}_{k}[Q] \subseteq \operatorname{Par}_{k}[Q]_{F} \subseteq \operatorname{Pos}_{k}[Q]_{F} \subseteq 1 \mathrm{~L}_{k}[Q]_{F} \subseteq 2 \mathrm{~L}_{k}[Q]_{F} \\
\operatorname{Par}_{k}[Q]_{R} \subseteq \operatorname{Pos}_{k}[Q]_{R} \subseteq 1 \mathrm{~L}_{k}[Q]_{R} \subseteq 2 \mathrm{~L}_{k}[Q]_{R}
\end{gathered}
$$

\section{3. Редукция формул}

Определим несколько правил эквивалентных преобразований формул в языках $1 \mathrm{~L}_{k}$ и $2 \mathrm{~L}_{k}$. Как обычно, формулу $\neg\left(t_{1}=t_{2}\right)$ будем записывать в виде $\left(t_{1} \neq t_{2}\right)$.

\section{1. Элиминирование квантора общности по предметной переменной}

Пусть $(\forall x) \Phi(x)$ - формула языка $2 \mathrm{~L}_{k}$, а предметные переменные $y_{1}, \ldots, y_{k}$ не входят в формулу $\Phi(x)$. Поскольку основное множество $E_{k}$ состоит из $k$ элементов, формула $(\forall x) \Phi(x)$ эквивалентна формуле

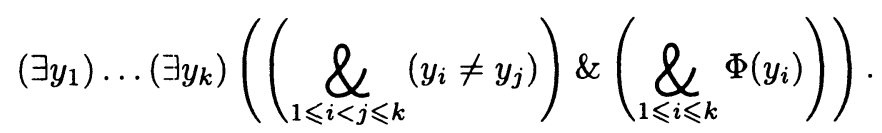

\section{2. Элиминирование квантора общности по функциональной переменной}

Идею преобразования формул из п. 1 применим для функциональных переменных. Пусть $\left(\forall F^{(n)}\right) \Phi\left(F^{(n)}\right)$ - формула языка $2 \mathrm{~L}_{k}, m=k^{k^{n}}$ и функциональные переменные $F_{1}^{(n)}, \ldots, F_{m}^{(n)}$ не входят в формулу $\Phi\left(F^{(n)}\right)$. Поскольку в $P_{k}$ имеется ровно 
$k^{k^{n}}$ функций, зависящих от $n$ переменных, формула $\left(\forall F^{(n)}\right) \Phi\left(F^{(n)}\right)$ эквивалентна формуле

$$
\left(\exists F_{1}^{(n)}\right) \ldots\left(\exists F_{m}^{(n)}\right)\left(\left(\bigotimes_{1 \leqslant i<j \leqslant m}\left(F_{i}^{(n)} \neq F_{j}^{(n)}\right)\right) \&\left(\bigotimes_{1 \leqslant i \leqslant m}\left(\Phi\left(F_{i}^{(n)}\right)\right)\right)\right)
$$

где $\left(F_{i}^{(n)} \neq F_{j}^{(n)}\right)$ есть сокращение для формулы

$$
\left(\exists x_{1}\right) \ldots\left(\exists x_{n}\right)\left(F_{i}^{(n)}\left(x_{1}, \ldots, x_{n}\right) \neq F_{j}^{(n)}\left(x_{1}, \ldots, x_{n}\right)\right) .
$$

\section{3. Приведение равенств к специальному виду}

Если $t_{1}, \ldots, t_{n}, t_{n+1}$ - термы языка $2 \mathrm{~L}_{k}$ и переменные $z_{1}, \ldots, z_{n}, z_{n+1}$ не входят в термы $t_{1}, \ldots, t_{n}, t_{n+1}$, то равенство $\left(F^{(n)}\left(t_{1}, \ldots, t_{n}\right)=t_{n+1}\right)$ эквивалентно формуле

$$
\left(\exists z_{1}\right) \ldots\left(\exists z_{n+1}\right)\left(\left(\bigotimes_{1 \leqslant i \leqslant n+1}\left(z_{i}=t_{i}\right)\right) \&\left(F^{(n)}\left(z_{1}, \ldots, z_{n}\right)=z_{n+1}\right)\right) .
$$

Аналогичная эквивалентность имеет место для случая, когда вместо функциональной переменной $F^{(n)}$ рассматривается функциональная константа $f^{(n)}$.

\section{4. Элиминирование квантора существования по функциональной переменной}

Пусть $\left(\exists F^{(n)}\right) \Phi\left(F^{(n)}\right)$ - формула языка $2 \mathrm{~L}_{k}$, в которой выполнены все возможные преобразования п. 3. Иными словами, пусть всякое равенство, входящее в формулу $\Phi\left(F^{(n)}\right)$, имеет либо вид $x_{i_{1}}=x_{i_{2}}$, либо вид $f_{p}^{(m)}\left(x_{i_{1}}, \ldots, x_{i_{m}}\right)=x_{i_{m+1}}$, либо вид $F_{q}^{(m)}\left(x_{i_{1}}, \ldots, x_{i_{m}}\right)=x_{i_{m+1}}$.

Предположим, что функция $\varphi\left(x_{1}, \ldots, x_{n}\right)$ из $P_{k}$ принимает $l$ значений $a_{1}, \ldots, a_{l}$, $1 \leqslant l \leqslant k$. Тогда существует разбиение множества $E_{k}^{n}$ на $l$ непустых попарно не пересекающихся подмножеств $X_{1}, \ldots, X_{l}$ такое, что на множестве $X_{i}$ функция $\varphi$ принимает значение $a_{i}, 1 \leqslant i \leqslant l$. Таким образом, существование функции $\varphi\left(x_{1}, \ldots, x_{n}\right)$ эквивалентно существованию числа $l, 1 \leqslant l \leqslant k$, упорядоченного набора $\left(a_{1}, \ldots, a_{l}\right)$ из $l$ попарно различных элементов множества $E_{k}$ и разбиения $\left(X_{1}, \ldots, X_{l}\right)$ множества $E_{k}^{n}$ на $l$ непустых попарно не пересекающихся подмножеств. Так как кванторы существования в этом утверждении распространяются по конечным множествам, их действие можно заменить соответствующей дизъюнкцией. Эта идея реализуется в следующей конструкции.

Для любого $l, 1 \leqslant l \leqslant k$, любого упорядоченного набора $\left(a_{1}, \ldots, a_{l}\right)$ из $l$ различных элементов, принадлежащих множеству $E_{k}$, и любого разбиения $\left(X_{1}, \ldots, X_{l}\right)$ множества $E_{k}^{n}$ на непустые попарно не пересекающиеся подмножества определяем формулу

$$
\left(\exists z_{0}^{1}\right) \ldots\left(\exists z_{k-1}^{1}\right) \ldots\left(\exists z_{0}^{n}\right) \ldots\left(\exists z_{k-1}^{n}\right)\left(\bigotimes_{1 \leqslant m \leqslant n} \underset{0 \leqslant i<j \leqslant k-1}{\ell}\left(z_{i}^{m} \neq z_{j}^{m}\right) \& \Phi^{\prime}\right)
$$

где переменные $z_{0}^{1}, \ldots, z_{k-1}^{n}$ не входят в формулу $\Phi\left(F^{(n)}\right)$, а формула $\Phi^{\prime}$ определяется по формуле $\Phi\left(F^{(n)}\right)$ следующим образом. Всякую подформулу формулы $\Phi\left(F^{(n)}\right)$ 
вида $F^{(n)}\left(x_{j_{1}}, \ldots, x_{j_{n}}\right)=x_{j_{n+1}}$ заменяем формулой

$$
\begin{aligned}
\left({ }_{(b, c, \ldots, d) \in X_{1}}\left(x_{j_{1}}=z_{b}^{1}\right) \&\left(x_{j_{2}}=z_{c}^{2}\right) \& \ldots \&\left(x_{j_{n}}=z_{d}^{n}\right) \supset\left(x_{j_{n+1}}=z_{a_{1}}^{1}\right)\right) \vee \ldots \\
\quad \vee\left(\bigvee_{(b, c, \ldots, d) \in X_{l}}\left(x_{j_{1}}=z_{b}^{1}\right) \&\left(x_{j_{2}}=z_{c}^{2}\right) \& \ldots \&\left(x_{j_{n}}=z_{d}^{n}\right) \supset\left(x_{j_{n+1}}=z_{a_{l}}^{1}\right)\right),
\end{aligned}
$$

где импликация つ стандартным образом определяется через связки $\vee, \neg$. Формула $\left(\exists F^{(n)}\right) \Phi\left(F^{(n)}\right)$ эквивалентна дизъюнкции всех формул (1), образованной для всех троек $l,\left(a_{1}, \ldots, a_{l}\right),\left(X_{1}, \ldots, X_{l}\right)$ указанного выше вида.

\section{5. Элиминирование функциональной переменной в формулах специального вида}

Пусть $\Phi\left(F^{(n)}\right)$ - формула языка $2 \mathrm{~L}_{k}$, которая содержит только одну свободную функциональную переменную $F^{(n)}$, а все предметные переменные в формуле $\Phi\left(F^{(n)}\right)$ связаны. Будем также предполагать, что в формуле $\Phi\left(F^{(n)}\right)$ выполнены все возможные преобразования п. 3. Пусть формула $\Phi\left(F^{(n)}\right)$ определяет функцию $\varphi\left(x_{1}, \ldots, x_{n}\right)$ из $P_{k}$. Обозначим через $\Psi\left(x_{1}, \ldots, x_{n}, x_{n+1}\right)$ формулу

$$
\left(\exists F^{(n)}\right)\left(\left(F^{(n)}\left(x_{1}, \ldots, x_{n}\right)=x_{n+1}\right) \& \Phi\left(F^{(n)}\right)\right) .
$$

В силу того, что формула $\Phi\left(F^{(n)}\right)$ определяет функцию $\varphi\left(x_{1}, \ldots, x_{n}\right)$, для любых $x_{1}, \ldots, x_{n}$ из $E_{k}$ существует единственное $x_{n+1}$ (именно, $x_{n+1}=\varphi\left(x_{1}, \ldots, x_{n}\right)$ ), для которого истинно значение $\Psi\left(x_{1}, \ldots, x_{n}, x_{n+1}\right)$. Поэтому, если формула $\Psi^{\prime}\left(x_{1}, \ldots, x_{n}, x_{n+1}\right)$ в языке $1 \mathrm{~L}_{k}$ получена из формулы $\Psi\left(x_{1}, \ldots, x_{n}, x_{n+1}\right)$ элиминированием кванторов общности и существования согласно пп. 2 и 4 , то формула $\Psi^{\prime}\left(x_{1}, \ldots, x_{n}, x_{n+1}\right)$ будет $1 \mathrm{~L}_{k}$-выражать функцию $\varphi\left(x_{1}, \ldots, x_{n}\right)$.

В заключение раздела рассмотрим вопрос о существовании языков, занимающих промежуточное положение между $\operatorname{Pos}_{k}$ и $1 \mathrm{~L}_{k}$. По определению, язык $1 \mathrm{~L}_{k}$ получается из языка $\operatorname{Pos}_{k}$ добавлением отрицания и квантора общности. Очевидно, мы получим язык, эквивалентный языку $1 \mathrm{~L}_{k}$, если добавим к языку $\operatorname{Pos}_{k}$ только одно отрицание. Оказываєтся, что такой же результат имеет место при добавлении к языку $\mathrm{Pos}_{k}$ только квантора общности. В самом деле, согласно пп. 1, 3 и правилам логики всякую формулу языка $1 \mathrm{~L}_{k}$ можно получить из формул вида

$$
x_{i_{1}}=x_{i_{2}}, \quad f_{j}^{(n)}\left(x_{i_{1}}, \ldots, x_{i_{n}}\right)=x_{i_{n+1}}
$$

и отрицаний этих формул с помощью применения операций $\&, \vee, \exists$. Поскольку формула

$$
\neg\left(f_{j}^{(n)}\left(x_{i_{1}}, \ldots, x_{i_{n}}\right)=x_{i_{n+1}}\right)
$$

эквивалентна формуле

$$
(\exists y)\left(\left(f_{j}^{(n)}\left(x_{i_{1}}, \ldots, x_{i_{n}}\right)=y\right) \& \neg\left(y=x_{i_{n+1}}\right)\right),
$$

все сводится к вопросу о том, имеются ли в языке Pos $_{k}$ с добавленным квантором общности формулы, эквивалентные формуле $x_{1} \neq x_{2}$. Легко видеть, что одной из 
таких формул будет формула

$$
\left(\exists y_{1}\right) \ldots\left(\exists y_{k}\right)(\forall z)\left(\left(\bigvee_{1 \leqslant i \leqslant k}\left(z=y_{i}\right)\right) \&\left(x_{1}=y_{1}\right) \&\left(x_{2}=y_{2}\right)\right) .
$$

Таким образом, в смысле выразимости не существует естественных языков, занимающих промежуточное положение между $\operatorname{Pos}_{k}$ и $1 \mathrm{~L}_{k}$.

\section{4. Основные результаты}

Применение пп. 2-5 к формулам языка $2 \mathrm{~L}_{k}$ приводит к следующему результату.

Теорема 1. Для любого $k, k \geqslant 2$, совокупности $1 \mathrm{~L}_{k}$-замкнутых $u 2 \mathrm{~L}_{k}$-замкнутых классов отношений (функций) совпадают. Более того, для любого множества $Q$, $Q \subseteq P_{k}$, имеют место равенства

$$
\begin{aligned}
1 \mathrm{~L}_{k}[Q]_{R} & =2 \mathrm{~L}_{k}[Q]_{R} \\
1 \mathrm{~L}_{k}[Q]_{F} & =2 \mathrm{~L}_{k}[Q]_{F}
\end{aligned}
$$

Обозначим через $1 \exists \mathrm{L}_{k}$ множество формул языка $1 \mathrm{~L}_{k}$, которые в предваренной нормальной форме не содержат кванторов общности. Из п. 1 вытекает, что при определении понятий выразимости и замыкания в языке $1 \mathrm{~L}_{k}$ можно ограничиться только формулами из $1 \exists \mathrm{L}_{k}$.

Предложение 1. Пусть $\mathrm{L}_{k}$ - один из языков $\operatorname{Par}_{k}, \operatorname{Pos}_{k}, 1 \mathrm{~L}_{k}$. Тогда мощность множества всех $\mathrm{L}_{k}$-замкнутых классов функций не превосходит мощности множества всех $\mathrm{L}_{k}$-замкнутых классов отношений.

Доказательство. Достаточно заметить, что формула $\Phi$ языка $\mathrm{L}_{k}$, определяющая функцию $\varphi\left(x_{1}, \ldots, x_{n}\right)$, одновременно определяет отношение $\varphi\left(x_{1}, \ldots, x_{n}\right)=y$. Таким образом, если $\mathrm{L}_{k}\left[Q_{1}\right]_{F} \neq \mathrm{L}_{k}\left[Q_{2}\right]_{F}$, то также $\mathrm{L}_{k}\left[Q_{1}\right]_{R} \neq \mathrm{L}_{k}\left[Q_{2}\right]_{R}$.

Для любого $k, k \geqslant 2$, обозначим через $\mathscr{S}_{k}$ полную симметрическую группу подстановок на множестве $E_{k}$. Следуя [30] (см. также [31, 33]), функцию $\varphi\left(x_{1}, \ldots, x_{n}\right)$ из $P_{k}$ назовем однородной, если она самодвойственна относительно любых подстановок группы $\mathscr{S}_{k}$. Таким образом, функция $\varphi\left(x_{1}, \ldots, x_{n}\right)$ однородна, если для любой подстановки $\pi$ из $\mathscr{S}_{k}$ имеет место тождество

$$
\varphi\left(\pi\left(x_{1}\right), \ldots, \pi\left(x_{n}\right)\right)=\pi\left(\varphi\left(x_{1}, \ldots, x_{n}\right)\right)
$$

Множество всех однородных функций из $P_{k}$ обозначим через $H_{k}$. Легко видеть, что множество $H_{k}$ замкнуто относительно операции суперпозиции.

Теорема 2. Для любого $k, k \geqslant 2$, имеет место равенство

$$
1 \mathrm{~L}_{k}[\varnothing]_{F}=H_{k}
$$


Доказателъство. При $k \geqslant 2$ определим на множестве $E_{k}$ однородные функции $p_{k}$ и $r_{k}$, полагая

$$
\begin{aligned}
p_{k}(x, y, z) & = \begin{cases}z, & \text { если } x=y, \\
x & \text { в противном случае }\end{cases} \\
r_{k}\left(x_{1}, \ldots, x_{k-1}\right) & = \begin{cases}x_{k}, & \text { если }\left\{x_{1}, \ldots, x_{k-1}, x_{k}\right\}=E_{k}, \\
x_{1} & \text { в противном случае. }\end{cases}
\end{aligned}
$$

При $k=2$ функции $p_{k}$ и $r_{k}$ совпадают с булевыми функциями $x \bar{y} \vee x z \vee \bar{y} z$ и $\bar{x}_{1}$. Хорошо известно, что функции $x \bar{y} \vee x z \vee \bar{y} z, \bar{x}_{1}$ образуют базис по суперпозиции в классе $H_{2}$, классе всех самодвойственных булевых функций (см. [6], где класс $H_{2}$ имеет обозначение $D_{3}$ ). Аналогично, при $k \geqslant 3$ базис в классе $H_{k}$ образуют функции $p_{k}, r_{k}$ (см. [32], а также [31, 33]). Поскольку всякий $1 \mathrm{~L}_{k}$-замкнутый класс функций замкнут относительно операции суперпозиции, для доказательства включения $H_{k} \subseteq 1 \mathrm{~L}_{k}[\varnothing]_{F}$ достаточно установить, что функции $p_{k}, r_{k}$ можно выразить формулами языка $1 \mathrm{~L}_{k}$, не содержащими символов функций. Для функции $p_{k}(x, y, z)$ такой формулой будет формула

$$
((x=y) \supset(v=z)) \&((x \neq y) \supset(v=x)),
$$

для функции $r_{k}\left(x_{1}, \ldots, x_{k-1}\right)$ - формула

$$
\left(\left(\exists x_{k}\right)\left(\bigotimes_{1 \leqslant i<j \leqslant k}\left(x_{i} \neq x_{j}\right)\right) \supset\left(y=x_{k}\right)\right) \&\left(\bigvee_{1 \leqslant i<j \leqslant k-1}\left(x_{i}=x_{j}\right) \supset\left(y=x_{1}\right)\right)
$$

(при $k=2$ последняя формула упрощается до $y \neq x_{1}$ ).

Покажем, что $1 \mathrm{~L}_{k}[\varnothing]_{F} \subseteq H_{k}$. С этой целью заметим, что группа автоморфизмов модели $\left\langle E_{k} ; \Rightarrow\right.$ совпадает с $\mathscr{S}_{k}$. Поэтому, если формула $\Phi\left(x_{1}, \ldots, x_{n}, y\right)$ языка $1 \mathrm{~L}_{k}$, не содержащая символов функций, определяет функцию $\varphi\left(x_{1}, \ldots, x_{n}\right)$, то группа автоморфизмов алгебры $\left\langle E_{k} ; \varphi\right\rangle$ будет также совпадать с $\mathscr{S}_{k}$ (см., например, [34], глава 3). Другими словами, функция $\varphi$ будет однородной. Теорема доказана.

Пусть $G$ - подгруппа группы $\mathscr{S}_{k}$. Обозначим через $P_{k} G$ множество всех функций из $P_{k}$, которые самодвойственны относительно любых подстановок группы $G$. Иначе говоря, $P_{k} G$ есть множество всех функций $\varphi$ из $P_{k}$ таких, что группа автоморфизмов алгебры $\left\langle E_{k} ; \varphi\right\rangle$ содержи' группу $G$. Таким образом, $P_{k} \mathscr{S}_{k}=H_{k}$ и $P_{k} e_{k}=P_{k}$, где $e_{k}$ - единичная подгруппа группы $\mathscr{S}_{k}$. Нетрудно убедиться в том, что для любой группы $G, G \subseteq \mathscr{S}_{k}$, множество $P_{k} G$ замкнуто относительно операции суперпозиции.

Пусть $G$ - подгруппа группы $\mathscr{S}_{k},\left(a_{1}, \ldots, a_{m}\right),\left(b_{1}, \ldots, b_{m}\right)$ - произвольные наборы из $E_{k}^{m}, m$ - натуральное число. Будем говорить, что $\left(a_{1}, \ldots, a_{m}\right)$ и $\left(b_{1}, \ldots, b_{m}\right)$ $G$-эквивалентны, если один из них можно перевести в другой с помощью некоторой подстановки группы $G$, т.е. если в группе $G$ существует такая подстановка $\pi$, что

$$
\left(b_{1}, \ldots, b_{m}\right)=\left(\pi\left(a_{1}\right), \ldots, \pi\left(a_{m}\right)\right)
$$

или

$$
\left(a_{1}, \ldots, a_{m}\right)=\left(\pi\left(b_{1}\right), \ldots, \pi\left(b_{m}\right)\right) .
$$

Очевидно, что $G$-эквивалентность является отношением эквивалентности на множестве $E_{k}^{m}$. Классы $G$-эквивалентных наборов из $E_{k}^{m}$ называются $m$-орбитами группы $G$. 
Лемма 1. Если $G_{1}, G_{2}$ - различные подгруппы группы $\mathscr{S}_{k}$, mo

$$
P_{k} G_{1} \neq P_{k} G_{2}
$$

Доказательство. Пусть, например, $G_{2} \backslash G_{1} \neq \varnothing$ и $\pi \in G_{2} \backslash G_{1}$. Поскольку подстановка $\pi$ полностью определяется набором $(\pi(0), \pi(1), \ldots, \pi(k-1))$ и $\pi \notin G_{1}$, наборы $(0,1, \ldots, k-1)$ и $(\pi(0), \pi(1), \ldots, \pi(k-1))$ принадлежат различным $k$-орбитам группы $G_{1}$. Отсюда следует, что значения $k$-местных функций из класса $P_{k} G_{1}$ на данных наборах можно определять произвольным образом. Вместе с тем $\pi \in G_{2}$ и, следовательно, упомянутые наборы принадлежат одной и той же $k$-орбите группы $G_{2}$. Предположим, что $\pi(0)=a$. Если $b \neq a$, то определим в классе $P_{k} G_{1}$ такую функцию $\varphi\left(x_{1}, \ldots, x_{k}\right)$, что

$$
\varphi(0,1, \ldots, k-1)=0, \quad \varphi(\pi(0), \pi(1), \ldots, \pi(k-1))=b
$$

(это можно сделать, поскольку подстановка $\pi$ отлична от тождественной). Тогда $\varphi \notin P_{k} G_{2}$, так как предположение $\varphi \in P_{k} G_{2}$ влечет равенство

$$
\varphi(\pi(0), \pi(1), \ldots, \pi(k-1))=a .
$$

Лемма доказана.

Теорема 3. При любом $k, k \geqslant 2$, множество $1 \mathrm{~L}_{k}$-замкнутых классов бункций совпадает с множеством классов вида $P_{k} G$, где $G$ - подгруппа группы $\mathscr{S}_{k}$.

Доказательство. Установим вначале, что всякий класс вида $P_{k} G$ является $1 \mathrm{~L}_{k}$ замкнутым. В самом деле, пусть функция $\varphi 1 \mathrm{~L}_{k}$-выразима через функции $\varphi_{1}, \ldots, \varphi_{m}$ из класса $P_{k} G$. Согласно включению $\left\{\varphi_{1}, \ldots, \varphi_{m}\right\} \subset P_{k} G$ и в силу очевидных свойств отношения равенства группа автоморфизмов алгебраической системы $\left\langle E_{k} ;=, \varphi_{1}, \ldots, \varphi_{m}\right\rangle$ содержит группу $G$. В этом случае, как хорошо известно (см. [34], глава 3), группа $G$ будет также подгруппой группы автоморфизмов алгебры $\left\langle E_{k} ; \varphi\right\rangle$. Это означает, что $\varphi \in P_{k} G$.

Таким образом, в силу леммы 1 число $1 \mathrm{~L}_{k}$-замкнутых классов функций не меньше числа всех подгрупп группы $\mathscr{S}_{k}$. С другой стороны, согласно предложению 1 число $1 \mathrm{~L}_{k}$-замкнутых классов функций не больше $1 \mathrm{~L}_{k}$-замкнутых классов отношений.

Определим язык $1 \mathrm{RL}_{k}$. Он отличается от языка $1 \mathrm{~L}_{k}$ только тем, что для всякого $n, n \geqslant 1$, вместо символов функций $f_{i}^{(n)}$ содержит символы $R_{1}^{(n)}, \ldots, R_{q(n)}^{(n)}$ всех $n$-местных отношений на $E_{k}\left(q(n)=2^{k^{n}}\right)$. Если $R_{s}^{(l+1)}, R_{t}^{(m+1)}, \ldots, R_{u}^{(n+1)}$ суть обозначения соответственно отношений $f_{a}^{(l)}\left(x_{1}, \ldots, x_{l}\right)=y, f_{b}^{(m)}\left(x_{1}, \ldots, x_{m}\right)=y, \ldots$, $f_{c}^{(n)}\left(x_{1}, \ldots, x_{n}\right)=y$, то формула языка $1 \mathrm{~L}_{k}$ вида

$$
f_{a}^{(l)}\left(f_{b}^{(m)}\left(x_{1}^{1}, \ldots, x_{m}^{1}\right), \ldots, f_{c}^{(n)}\left(x_{1}^{l}, \ldots, x_{n}^{l}\right)\right)=y
$$

определяет то же самое отношение, что и формула

$$
\begin{aligned}
& \left(\exists y_{1}\right) \ldots\left(\exists y_{l}\right)\left(R_{t}^{(m+1)}\left(x_{1}^{1}, \ldots, x_{m}^{1}, y_{1}\right)\right. \\
& \& \ldots \\
& \left.\& R_{u}^{(n+1)}\left(x_{1}^{l}, \ldots, x_{n}^{l}, y_{l}\right) \& R_{s}^{(l+1)}\left(y_{1}, \ldots, y_{l}, y\right)\right)
\end{aligned}
$$


языка $1 \mathrm{~L}_{k}$. Отсюда сразу следует, что число $1 \mathrm{~L}_{k}$-замкнутых классов отношений не превосходит числа $1 \mathrm{RL}_{k}$-замкнутых классов отношений. Однако $1 \mathrm{RL}_{k}$-замкнутый класс отношений в терминологии работы [35] представляет собой алгебру Краснера второго рода. В [35] установлено, что число алгебр Краснера второго рода на множестве $E_{k}$ равно числу подгрупп симметрической группы $\mathscr{S}_{k}$. Следовательно, число $1 \mathrm{~L}_{k}$-замкнутых классов функций равно числу подгрупп группы $\mathscr{S}_{k}$, и теорема доказана.

Из теоремы 3 вытекает, что множество всех $1 \mathrm{~L}_{k}$-замкнутых классов функций (а также отношений) антиизоморфно (по отношению включения) решетке подгрупп симметрической группы $\mathscr{S}_{k}$. Это позволяет легко определить все $1 \mathrm{~L}_{k}$-предполные классы в $P_{k}$ и тем самым решить проблему полноты для $1 \mathrm{~L}_{k}$-выразимости.

Следствие 1. Класс $P_{k} G$ являетсл $1 \mathrm{~L}_{k}$-предполнъм в $P_{k}$ тогда и только тогда, когда группа $G$ порожсается неединичной подстановкой, цикловое разложение которой состоит из одноэлементных циклов и циклов одной и той же простой длинь.

Дожазательство. В силу антиизоморфизма между решеткой $1 \mathrm{~L}_{k}$-замкнутых классов функций и решеткой подгрупп группы $\mathscr{S}_{k}$ класс $P_{k} G$ будет $1 \mathrm{~L}_{k}$-предполным в том и только том случае, когда группа $G$ не содержит собственных подгрупп, т.е. когда $G$ задает атом в решетке подгрупп симметрической группы $\mathscr{S}_{k}$. Отсюда легко выводится утверждение следствия.

В качестве примера рассмотрим случаи $k=2,3,4$. В группе $\mathscr{S}_{2}$ имеются две подгруппы, $\mathscr{S}_{2}$ и единичная подгруппа $e_{2}$. Соответствующими $1 \mathrm{~L}_{2}$-замкнутыми классами функций будут класс $H_{2}$ всех однородных функций (класс $D_{3}$ всех самодвойственных булевых функций [6]) и класс $P_{2}$. В группе $\mathscr{S}_{3}$ имеется 6 подгрупп. Из них атомы определяют 4 подгруппы, отличные от $\mathscr{S}_{3}$ и $e_{3}$. Эти подгруппы порождаются подстановками с цикловыми разложениями

$$
(0)(12), \quad(01)(2), \quad(02)(1), \quad(012) \text {. }
$$

В решетке подгрупп группы $\mathscr{S}_{4}$ атомы определяются 13 подгруппами, которые порождаются подстановками

$$
\begin{array}{cccc}
(01)(2)(3), & (02)(1)(3), & (03)(1)(2), & (0)(12)(3), \\
(0)(13)(2), \quad(0)(1)(23), & (01)(23), & (02)(13), \\
(03)(12), \quad(012)(3), & (013)(2), & (023)(1), & (0)(123) .
\end{array}
$$

Функция $\varphi\left(x_{1}, \ldots, x_{n}\right)$ из $P_{k}$ называется селекторной, если для некоторого $i$, $1 \leqslant i \leqslant n$, выполняется тождество

$$
\varphi\left(x_{1}, \ldots, x_{i}, \ldots, x_{n}\right)=x_{i} .
$$

Обозначим через $D_{1}$ класс всех самодвойственных булевых $\alpha$-функций (см. [6]).

Теорема 4. При $k \geqslant 3$ класс $\operatorname{Pos}_{k}[\varnothing]_{F}$ состоит толъко из селекторных функций, $\operatorname{Pos}_{2}[\varnothing]_{F}=D_{1}$. 
Доказательство. Пусть $k \geqslant 2$. Приведем формулы языка $\operatorname{Pos}_{k}$, не содержащие символов функций, к специальному виду. Для этого воспользуемся тремя хорошо известными логическими эквивалентностями:

$$
\begin{aligned}
(\exists x)(\Phi \vee \Psi) & \equiv(\exists x) \Phi \vee(\exists x) \Psi, \\
(\exists x)(\Phi \& \Psi) & \equiv \Phi \&(\exists x) \Psi, \\
(\exists x)\left(\&_{1 \leqslant i \leqslant n}\left(x=x_{i}\right)\right) & \equiv \&_{1 \leqslant i<j \leqslant n}\left(x_{i}=x_{j}\right), \quad n \geqslant 2
\end{aligned}
$$

(при $n=1$ левая часть последней эквивалентности тождественно истинна), где переменная $x$ во второй эквивалентности не входит в формулу $\Phi$ свободно. Применяя эти эквивалентности и пользуясь дистрибутивностью конъюнкции относительно дизъюнкции, всякую не тождественно истинную формулу языка $\operatorname{Pos}_{k}$, не содержащую символов функций, можно привести к виду

$$
\Phi_{1} \vee \ldots \vee \Phi_{m}
$$

где каждая формула $\Phi_{i}$ является конъюнкцией равенств некоторых переменных.

Пусть теперь $k=2$. Как известно [6], базис по суперпозиции в классе $D_{1}$ образует функция

$$
p_{2}(x, y, z)=x \bar{y} \vee x z \vee \bar{y} z
$$

Поскольку формула

$$
(x=y) \&(v=z) \vee(x=z) \&(v=x) \vee(y=z) \&(v=x)
$$

языка $\mathrm{Pos}_{2}$, не содержащая символов функций, выражает функцию $p_{2}(x, y, z)$, мы имеем включение $D_{1} \subseteq \operatorname{Pos}_{2}[\varnothing]_{F}$. С другой стороны, очевидно, $\operatorname{Pos}_{2}[\varnothing]_{F} \subseteq 1 \mathrm{~L}_{2}[\varnothing]_{F}$. Как установлено в теореме $2,1 \mathrm{~L}_{2}[\varnothing]_{F}$ совпадает с классом $D_{3}$ всех самодвойственных булевых функций. Однако между $D_{1}$ и $D_{3}$ нет других замкнутых (относительно операции суперпозиции) классов [6]. Поэтому, если $\operatorname{Pos}_{2}[\varnothing]_{F} \neq D_{1}$, то $\operatorname{Pos}_{2}[\varnothing]_{F}=D_{3}$ и, следовательно, в $\operatorname{Pos}_{2}[\varnothing]_{F}$ входит функция $\bar{x}$. Предположим, что формула $\Phi(x, y)$ языка $\operatorname{Pos}_{2}$ выражает функцию $\bar{x}$. По доказанному можно считать, что формула $\Phi$ имеет вид $\Phi_{1} \vee \ldots \vee \Phi_{m}$, где каждая из формул $\Phi_{i}$ есть конъюнкция равенств. Опуская в формуле $\Phi_{1} \vee \ldots \vee \Phi_{m}$ конъюнктивные сомножители $x=x, y=y$ и равные дизъюнктивные слагаемые, приходим к выводу, что формула $\Phi(x, y)$ может иметь лишь вид $x=y$. Противоречие показывает, что $\operatorname{Pos}_{2}[\varnothing]_{F} \neq D_{3}$, и потому $\operatorname{Pos}_{2}[\varnothing]_{F}=D_{1}$.

Предположим, что $k \geqslant 3$. Легко убедиться в том, что классу $\operatorname{Pos}_{k}[\varnothing]_{F}$ принадлежат все селекторные функции. В самом деле, если $\varphi\left(x_{1}, \ldots, x_{i}, \ldots, x_{n}\right)$ - селекторная функция, совпадающая с $x_{i}$, то функцию $\varphi$ выражает формула

$$
\left(\bigotimes_{1 \leqslant j \leqslant n}\left(x_{j}=x_{j}\right)\right) \&\left(y=x_{i}\right)
$$

языка $\operatorname{Pos}_{k}$, не содержащая символов функций (множители $x_{j}=x_{j}$ указывают лишь на то, что функция $\varphi$ рассматривается от переменных $\left.x_{1}, \ldots, x_{n}\right)$.

Допустим, что класс $\operatorname{Pos}_{k}[\varnothing]_{F}$ содержит функцию $\varphi\left(x_{1}, \ldots, x_{n}\right)$, отличную от селекторной. И пусть функцию $\varphi\left(x_{1}, \ldots, x_{n}\right)$ выражает формула $\Phi\left(x_{1}, \ldots, x_{n}, y\right)$ языка $\operatorname{Pos}_{k}$, не содержащая символов функций. Будем считать, что формула $\Phi$ имеет 
вид $\Phi_{1} \vee \ldots \vee \Phi_{m}$, где каждая из формул $\Phi_{i}$ представляет собой конъюнкцию равенств некоторых переменных. Так как функция $\Phi$ отлична от селекторной и для любых значений переменных $x_{1}, \ldots, x_{n}$ имеется единственное значение переменной $y$, для которого истинно $\Phi\left(x_{1}, \ldots, x_{n}, y\right)$, каждая из конъюнкций $\Phi_{1}, \ldots, \Phi_{m}$ содержит хотя бы один сомножитель вида $x_{r}=x_{s}$, где $r \neq s$.

Из теоремы 2 следует, что функция $\varphi$ однородна. Согласно [31, 33] всякий замкнутый (относительно операции суперпозиции) класс однородных функций, отличный от класса селекторных функций, содержит при $k=3$ хотя бы одну из функций $d, l_{3}, 2 x_{1}+2 x_{2}$, при $k=4$ хотя бы одну из функций $d, l_{4}, x_{1}+x_{2}+x_{3}$ и при $k \geqslant 5$ хотя бы одну из функций $d, l_{k}$, где

$$
\begin{aligned}
d\left(x_{1}, x_{2}, x_{3}\right) & = \begin{cases}x_{1}, & \text { если } x_{1}=x_{2}, \\
x_{3} & \text { в противном случае, }\end{cases} \\
l_{k}\left(x_{1}, \ldots, x_{k}\right) & = \begin{cases}x_{1}, & \text { если } x_{1}, \ldots, x_{k} \text { попарно различны, } \\
x_{k} & \text { в противном случае }\end{cases}
\end{aligned}
$$

функция $2 x_{1}+2 x_{2}$ рассматривается в поле Галуа GF(3), а функция $\left(x_{1}+x_{2}+x_{3}\right)$ в поле Галуа GF(4). Таким образом, функцию $\varphi$ можно считать совпадающей с одной из функций $d, l_{k}, 2 x_{1}+2 x_{2}, x_{1}+x_{2}+x_{3}$. В частности, можно предполагать, что выполняется неравенство $n \leqslant k$. Однако в этом случае при $x_{1}=0, \ldots, x_{n}=n-1$ каждая из конъюнкций $\Phi_{1}, \ldots, \Phi_{m}$ становится ложной (за счет сомножителей вида $\left.x_{r}=x_{s}\right)$. Это противоречие завершает доказательство теоремы.

Пусть $\rho\left(x_{1}, \ldots, x_{m}\right)$ - отношение на $E_{k}$. Говорят, что функция $f\left(x_{1}, \ldots, x_{n}\right)$ из $P_{k}$ сохраняет отношение $\rho$, если для любых $n$ наборов $\left(a_{11}, \ldots, a_{m 1}\right), \ldots,\left(a_{1 n}, \ldots, a_{m n}\right)$, удовлетворяющих отношению $\rho$, набор $\left(f\left(a_{11}, \ldots, a_{1 n}\right), \ldots, f\left(a_{m 1}, \ldots, a_{m n}\right)\right)$ также удовлетворяет отношению $\rho$. Иными словами, функция $f$ сохраняет отношение $\rho$, если отношение $\rho$, рассматриваемое как подмножество множества $E_{k}^{m}$, образует подалгебру алгебры $\left\langle E_{k} ; f\right\rangle^{m}$. Через Pol $\rho$ обозначим множество всех функций из $P_{k}$, сохраняющих отношение $\rho$. Если $R$ - множество отношений, то пусть

$$
\operatorname{Pol} R=\bigcap_{\rho \in R} \operatorname{Pol} \rho
$$

Хорошо известно [35], что для любого множества отношений $R$ множество Pol $R$ является замкнутым (относительно операции суперпозиции) классом, содержащим все селекторные функции.

Пусть $\mathscr{T}_{k}$ есть симметрическая полугруппа всех отображений множества $E_{k}$ в себя. Если $T$ - подполугруппа полугруппы $\mathscr{T}_{k}$, то через $\operatorname{Gr}(T)$ обозначаем совокупность графиков всех функций из $T$, т.е. совокупность всех отношений вида

$$
\pi(x)=y,
$$

где $\pi \in T$.

Лемма 2. Для любого $k, k \geqslant 2, u$ любой подполугруппы $T$ полугруппы $\mathscr{T}_{k}$ класс $\operatorname{Pol} \operatorname{Gr}(T)$ является $\operatorname{Pos}_{k}$-замкнутым. 
Доказательство. Пусть формула $\Phi\left(x_{1}, \ldots, x_{n}, y\right)$ языка $\operatorname{Pos}_{k}$ выражает функцию $\varphi\left(x_{1}, \ldots, x_{n}\right)$ через функции множества $\operatorname{Pol} \operatorname{Gr}(T)$. Выполним в формуле $\Phi$ все возможные преобразования п. 3, приведем ее к пренексному виду и преобразуем бескванторную часть в дизъюнктивную нормальную форму. Вновь распределяя кванторы существования по дизъюнктивным слагаемым, будем предполагать, что формула $\Phi$ представима в виде $\Phi_{1} \vee \ldots \vee \Phi_{m}$, где каждое из дизъюнктивных слагаемых $\Phi_{i}$ представляет собой формулу

$$
\left(\exists z_{1}\right) \ldots\left(\exists z_{r_{i}}\right)\left(\Phi_{i 1} \& \ldots \& \Phi_{i s_{i}}\right)
$$

в которой каждая из подформул $\Phi_{i 1}, \ldots, \Phi_{i s_{i}}$ является равенством вида $u=v$ или

$$
f_{j}^{(l)}\left(u_{1}, \ldots, u_{l}\right)=v \quad\left(\left\{u, u_{1}, \ldots, u_{l}, v\right\} \subseteq\left\{x_{1}, \ldots, x_{n}, y, z_{1}, \ldots, z_{r_{i}}\right\}\right) .
$$

Допустим теперь, что $\varphi\left(a_{1}, \ldots, a_{n}\right)=b$ и на наборе $\left(a_{1}, \ldots, a_{n}, b\right)$ истинна формула (3). И пусть $c_{1}, \ldots, c_{r_{i}}-$ значения переменных $z_{1}, \ldots, z_{r_{i}}$ в формуле (3), обеспечивающие ее истинность при $x_{1}=a_{1}, \ldots, x_{n}=a_{n}, y=b$. Если $\pi \in T$, а функция $f_{j}^{(l)}$ из $P_{k}$ сохраняет отношение $(2)$, то по определению имеет место тождество

$$
f_{j}^{(l)}\left(\pi\left(y_{1}\right), \ldots, \pi\left(y_{l}\right)\right)=\pi\left(f_{j}^{(l)}\left(y_{1}, \ldots, y_{l}\right)\right) .
$$

Следовательно, если в формуле $\Phi_{i 1} \& \ldots \& \Phi_{i s_{i}}$ заменить значения $a_{1}, \ldots, a_{n}, b$, $c_{1}, \ldots, c_{r_{i}}$ соответственно значениями $\pi\left(a_{1}\right), \ldots, \pi\left(a_{n}\right), \pi(b), \pi\left(c_{1}\right), \ldots, \pi\left(c_{r_{i}}\right)$, то она останется истинной. Значит, формула (3) будет истинной при замене набора $\left(a_{1}, \ldots, a_{n}, b\right)$ набором $\left(\pi\left(a_{1}\right), \ldots, \pi\left(a_{n}\right), \pi(b)\right)$. Иными словами, будет справедливо равенство

$$
\varphi\left(\pi\left(a_{1}\right), \ldots, \pi\left(a_{n}\right)\right)=\pi(b) .
$$

Поскольку $\varphi\left(a_{1}, \ldots, a_{n}\right)=b$, приходим к выводу, что функция $\varphi$ сохраняет отношение (2). Лемма доказана.

Замечание 1. Не сужая совокупности $\operatorname{Pos}_{k}$-замкнутых классов, в лемме 2 можно рассматривать лишь полугруппы $T$ с единицей $\varepsilon$ (тождественным отображением). В самом деле, графиком отображения $\varepsilon$ является отношение $x=y$. Как известно [35], $\operatorname{Pol}\{x=y\}=P_{k}$. Поэтому для любой полугруппы $T$

$$
\operatorname{Pol} \operatorname{Gr}(T)=\operatorname{Pol} \operatorname{Gr}(T \cup\{\varepsilon\}) .
$$

Замечание 2. Если $T_{1}, T_{2}$ - различные подполугруппы полугруппы $\mathscr{T}_{k}$, содержащие единицу, то неравенство $\operatorname{Pol} \operatorname{Gr}\left(T_{1}\right) \neq \operatorname{Pol} \operatorname{Gr}\left(T_{2}\right)$, вообще говоря, не выполняется. В качестве примера рассмотрим в полугруппе $\mathscr{T}_{3}$ полугруппы $T_{1}, T_{2}$, порождаемые соответственно элементами $\varepsilon, \alpha, \beta$ и $\varepsilon, \alpha, \beta, \gamma$, где

$$
\varepsilon=\left(\begin{array}{lll}
0 & 1 & 2 \\
0 & 1 & 2
\end{array}\right), \quad \alpha=\left(\begin{array}{lll}
0 & 1 & 2 \\
0 & 0 & 2
\end{array}\right), \quad \beta=\left(\begin{array}{lll}
0 & 1 & 2 \\
2 & 0 & 2
\end{array}\right), \quad \gamma=\left(\begin{array}{lll}
0 & 1 & 2 \\
1 & 1 & 2
\end{array}\right) .
$$

Справедливо включение $T_{1} \subset T_{2}$, причем $\gamma \notin T_{1}$, поскольку $\gamma$ принимает лишь значения 1 и 2 . Покажем, что $\operatorname{Pol} \operatorname{Gr}\left(T_{1}\right)=\operatorname{Pol} \operatorname{Gr}\left(T_{2}\right)$. Согласно [35], это равенство будет выполняться, если график функции $\gamma$ выражается через графики функций $\varepsilon, \alpha, \beta$ с помощью логических формул, содержащих конъюнкцию и квантор существования. Положим

$$
\rho_{1}(x, y) \equiv(\exists z)((\alpha(x)=z) \&(\alpha(y)=z))
$$


Тогда

$$
\rho_{1}(x, y) \equiv((x, y \in\{0,1\}) \vee(x=y=2))
$$

Если теперь положить

$$
\rho_{2}(x, y) \equiv(\exists z)((\alpha(x)=z) \&(\beta(y)=z))
$$

TO

$$
\rho_{2}(x, y) \equiv(x \in\{0,1\}) \&(y=1) \vee(x=2) \&(y \in\{0,2\})
$$

и, следовательно,

$$
\rho_{1}(x, y) \& \rho_{2}(x, y) \equiv(\gamma(x)=y)
$$

На основе указанного примера можно, разумеется, построить аналогичные примеры при любом $k, k \geqslant 4$.

Теорема 5. При любом $k, k \geqslant 2$, иисло $\operatorname{Pos}_{k}$-замкнутых классов функций больше числа подгрупп симметрической группы $\mathscr{S}_{k} u$ не превосходит числа подполугрупп симметрической полугруппљ $\mathscr{T}_{k}$, содержащих единицу.

Доказателъство. Поскольку любой $1 \mathrm{~L}_{k}$-замкнутый класс функций является также $\mathrm{Pos}_{k}$-замкнутым, из теоремы 3 следует, что число $\operatorname{Pos}_{k}$-замкнутых классов функций не меньше числа подгрупп симметрической группы $\mathscr{S}_{k}$. Пусть $T_{0}-$ подполугруппа полугруппы $\mathscr{T}_{k}$, состоящая из одной функции-константы 0. Согласно лемме 2 класc $\mathrm{Pol} \operatorname{Gr}\left(T_{0}\right)$ является $\mathrm{Pos}_{k}$-замкнутым. Нетрудно видеть, что он состоит из всех функций класса $P_{k}$, сохраняющих константу 0. В частности, класс $\operatorname{Pol} \operatorname{Gr}\left(T_{0}\right)$ отличен от всех классов вида $P_{k} G$, где $G-$ подгруппа группы $\mathscr{S}_{k}$. Значит, число $\operatorname{Pos}_{k^{-}}$ замкнутых классов функций больше числа подгрупп симметрической группы $\mathscr{S}_{k}$.

При доказательстве верхней оценки действуем по той же схеме, что и в доказательстве теоремы 3. Именно, используя предложение 1 , заключаем, что число $\mathrm{Pos}_{k}$-замкнутых классов функций не превосходит числа $\operatorname{Pos}_{k}$-замкнутых классов отношений. Аналогично языку $1 \mathrm{RL}_{k}$ из доказательства теоремы 3 вводим язык $\mathrm{RPos}_{k}$, заменяя в языке $\mathrm{Pos}_{k}$ символы функций символами отношений на $E_{k}$. Как и в теореме 3 , убеждаемся, что число $\operatorname{Pos}_{k}$-замкнутых классов отношений не превосходит числа $\operatorname{RPos}_{k}$-замкнутых классов отношений. Согласно терминологии работы [35] $\mathrm{RPos}_{k}$-замкнутый класс отношений есть алгебра Краснера первого рода. В [35] доказано, что число алгебр Краснера первого рода на множестве $E_{k}$ равно числу подполугрупп симметрической полугруппы $\mathscr{T}_{k}$, содержащих единицу. Следовательно, число $\mathrm{Pos}_{k}$-замкнутых классов отношений не превосходит числа подполугрупп полугруппы $\mathscr{T}_{k}$, содержацих единицу. Теорема доказана.

Замечание 3. В булевом случае число Pos$_{2}$-замкнутых классов функций равно числу подполугрупп полугруппы $\mathscr{T}_{2}$, содержащих единицу, а каждый $\operatorname{Pos}_{2}$-замкнутый класс определяется в соответствии с леммой 2 и замечанием 1 . В самом деле, имеется ровно 6 подполугрупп полугруппы $\mathscr{T}_{2}$, содержащих единицу (функцию $x$ ):

$$
\{x\},\{x, 0\}, \quad\{x, 1\}, \quad\{x, 0,1\}, \quad\{x, \bar{x}\}, \quad\{x, \bar{x}, 0,1\} .
$$

При отображении $\operatorname{Pol} \operatorname{Gr}(T)$ им соответствуют шесть $\mathrm{Pos}_{2}$-замкнутых классов булевых функций: $P_{2}, C_{3}, C_{2}, C_{4}, D_{3}, D_{1}$ (обозначения классов взяты из [6]). 
В заключение сравним операторы замыкания (на множестве функций), отвечающие языкам $0 \mathrm{~L}_{k}, \operatorname{Par}_{k}, \operatorname{Pos}_{k}, 1 \mathrm{~L}_{k}$. Как известно [4-6], имеется счетное число $0 \mathrm{~L}_{2}$ замкнутых классов. А.В. Кузнецовым [23] установлено, что число $\mathrm{Par}_{2}$-замкнутых классов равно 25. При $k \geqslant 3$ число $0 \mathrm{~L}_{k}$-замкнутых классов континуально [10], а число $\operatorname{Par}_{k}$-замкнутых классов конечно $[24,29]$. Далее, при переходе от языка $\operatorname{Par}_{2}$ к языку $\mathrm{Pos}_{2}$ число соответствующих замкнутых классов уменьшается с 25 до 6 , а при переходе от языка $\operatorname{Pos}_{2}$ к языку $1 \mathrm{~L}_{2}$ с 6 до 2 . Кроме того, при $k \geqslant 3$ число $\operatorname{Pos}_{k}$-замкнутых классов строго больше числа $1 \mathrm{~L}_{k}$-замкнутых классов. Эти результаты показывают, что увеличение выразительных возможностей языка в цепочке $0 \mathrm{~L}_{k} \subset \operatorname{Par}_{k} \subset \operatorname{Pos}_{k} \subset 1 \mathrm{~L}_{k}$ приводит к строгому усилению соответствующих операторов замыкания.

\section{Список литературы}

1. Яблонский С.В., Функциональные построения в $k$-значной логике. Труды Матем. инта АН СССР им. В.А. Стеклова (1958) 51, 5-142.

2. Яблонский С.В., Введение в теорию функций $k$-значной логики. Дискретная математика и математические вопросы кибернетики. Наука, Москва, 1974, 9-66.

3. Кудрявцев В.Б., Функциональная система. Матем. энциклопедия, т.5. Советская энциклопедия, Москва, 1985, 694-696.

4. Post E.L., Introduction to a general theory of elementary propositions. Amer. J. Math. (1921) 43, 163-185.

5. Post E.L., Two-valued iterative systems of mathematical logic. Ann. Math. Studies (1941) 5.

6. Яблонский С.В., Гаврилов Г.П., Кудрявцев В.Б., Функции алгебры логики и классъ Поста. Наука, Москва, 1966.

7. Яблонский С.В., О функциональной полноте в трехзначном исчислении. Докл. $A H$ CCCP (1954) 95, №6, 1153-1156.

8. Rosenberg I., La structure des fonctions de plusieurs variables sur un ensemble fini. C. R. Acad. Sci. Paris, Ser. A-B (1965) 260, №14, 3817-3819.

9. Rosenberg I.G., Über die funktionale Vollständigkeit in den mehrwertigen Logiken. Rozpravy Československe Akad. Vĕd ̌̆ada Math. Přir. Vĕd. (1970) 80, 3-93.

10. Янов Ю.И., Мучник А.А., О существовании $k$-значных замкнутых классов, не имеющих конечного базиса. Докл. АН ССCP (1959) 127, №1, 44-46.

11. Нгуен Ван Хоа, О структуре самодвойственных замкнутых классов трехзначной логики $P_{3}$. Дискретная математика (1992) 4, №4, 82-95.

12. Нгуен Ван Хоа, О семействах замкнутых классов $k$-значной логики, сохраняемых всеми автоморфизмами. Дискретная математика (1993) 5, №4, 87-108.

13. Нгуен Ван Хоа, Описание замкнутых классов, сохраняемых всеми внутренними автоморфизмами. Докл. АН Беларуси (1994) 38, №3, 16-19.

14. Нгуен Ван Хоа, О структуре замкнутых классов $k$-значной логики, самодвойственных относительно транзитивных групп. Докл. АН Беларуси (1994) 38, №6, 17-20.

15. Нгуен Ван Хоа, О замкнутых классах $k$-значной логики, самодвойственных относительно транзитивных групп. Дискретная математиха (1996) 8, №1, 129-156.

16. Марченков С.С., Основные отношения $S$-классификации функций многозначной логики. Дискретная математиха (1996) 8, №1, 99-128. 
17. Марченков С.С., $S$-классификация идемпотентных алгебр с конечным носителем. Докл. РАН (1996) 348, №5, 587-589.

18. Марченков C.C., G-предполные классы многозначной логики. Дискретный анализ $u$ исследование операций (1996) 3, №3, 47-70.

19. Марченков С.С., $S$-классификация функций многозначной логики. Дискретная математика (1997) 9, №3, 125-152.

20. Марченков С.С., $A$-классификация конечных инъективных функций. Дискретный анализ и исследование операчий (1997) 4, №2, 15-42.

21. Марченков С.С., $A$-замкнутые классы идемпотентных функций многозначной логики, определяемые двуместными отношениями. Дискретный анализ и исследование операций (1998) 5, №1, 32-59.

22. Марченков С.С., $A$-замкнутые классы многозначной логики, содержащие константы. Дискретная математика (1998) 10, №3, 10-26.

23. Кузнецов А.В., О средствах для обнаружения невыводимости и невыразимости. В кн.: Логический вывод. Наука, Москва, 1979, с. 5-33.

24. Данильченко А.Ф., О параметрической выразимости функций трехзначной логики. $А \Omega$ гебра и логика (1977) 16, №4, 397-416.

25. Раца М.Ф., Выразимость в исчислениях высказываний. Штиинца, Кишинев, 1991.

26. Голунков Ю.В., Полнота систем функций в операторных алгоритмах, реализующих функции $k$-значной логики. Вероятностные методы и кибернетика (1980) 17, 23-34.

27. Тайманов В.А., О функциональных системах $k$-значной логики с операциями программного типа. Докл. АН СССР (1983) 268, №6, 1307-1310.

28. Соловьев В.Д., Замкнутые классы в $k$-значной логике с операцией разветвления по предикату. Дискретная математика (1990) 2, №4, 19-25.

29. Burris S., Willard R., Finitely many primitive positive clones. Proc. Amer. Math. Soc. (1987) 101, №3, 427-430.

30. Marczewski E., Homogeneous operations and homogeneous algebras. Fund. Math. (1964) 56, №2, 81-103.

31. Марченков С.С., О зам.кнутых классах самодвойственных функций многозначной логики. Проблемъ кибернетики (1979) 36, 5-22.

32. Csákány B., Gavalcová 'T., Finite homogeneous algebras. Acta Sci. Math (1980) 42, №1-2, $57-65$.

33. Марченков С.С., Однородные алгебры. Проблемы кибернетики (1982) 39, 85-106.

34. Мальцев А.И., Алгебраические системы. Наука, Москва, 1970.

35. Боднарчук В.Г., Калужнин Л.А., Котов В.Н., Ромов Б.А., Теория Галуа для алгебр Поста. Кибернетика (1969) 3, 1-10; 5, 1-9.

Статья поступила 05.11.1998. 